\title{
Characterization and Implementation of in-to-out Body Wireless Sensor Data Transmission for Smaller Ruminants ${ }^{+}$
}

\author{
Lu Wang 1,*, Günter Vermeeren ${ }^{2}$, Marta Prim ${ }^{1}$, Wout Joseph ${ }^{2}$ and Carles Ferrer ${ }^{1}$ \\ 1 Department of Microelectronics and Electronic Systems, Autonomous University of Barcelona, \\ 08193 Bellaterra, Spain; marta.prim@uab.es (M.P.); carles.ferrer@uab.es (C.F.) \\ 2 Department of Information Technology (INTEC), Ghent University/IMEC, 9052 Ghent, Belgium; \\ gunter.vermeeren@ugent.be (G.V.); wout.joseph@ugent.be (W.J.) \\ * Correspondence: lu.wang@uab.es \\ + Presented at the 5th International Symposium on Sensor Science (I3S 2017), Barcelona, Spain, \\ 27-29 September 2017.
}

Published: 6 December 2017

Among bioclimatic indicators for ruminants, the rumen stability is crucial to determine the animal's health status. A modern solution to monitor the rumen condition consists of an implant sensing bolus that collects and transmits bioclimatic ruminal data, such as temperature and $\mathrm{pH}$ value, and a wearable on-body receiver which also communicates with a remote control platform. Such a solution is being adopted in cattle management as the bovine animal size does not impose strict requirements on the dimensions and weight of the bolus. However, for smaller ruminants such as sheep and goats, research on autonomous health monitoring and ruminal sensing bolus development is lagging behind. In this work, we characterized numerically the in-to-out body wireless data transmission in smaller ruminants using the 3D electromagnetic finite-difference time-domain (FDTD) solver available in sim4life (ZMT, Zurich, Switzerland). First, we dimensioned a spiral antenna operating at $433 \mathrm{MHz}$ for integration in a ruminal bolus whose volume is $70 \%$ less than that of a bovine bolus while taking into account the frequency detuning due to the proximity of the printed circuit board (PCB) and animal tissues. Next, we investigated the in-to-out body path loss in a homogeneous animal model and verified it with a bioequivalent phantom. The in-to-out body path loss analysis can be used to predict in vivo propagation with living adult sheep or goats.

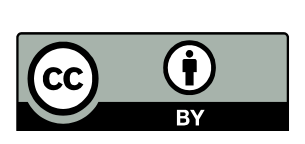

(C) 2017 by the authors. Licensee MDPI, Basel, Switzerland. This article is an open access article distributed under the terms and conditions of the Creative Commons Attribution (CC BY) license (http://creativecommons.org/licenses/by/4.0/). 